\title{
Application of Reflected Global Navigation Satellite System (GNSS-R) Signals in the Estimation of Sea Roughness Effects in Microwave Radiometry
}

\author{
Justin K. Voo ${ }^{*}$, James L. Garrison*, Simon H. Yueh ${ }^{\ddagger}$, Michael S. Grant ${ }^{\S}$, Alexander G. Fore ${ }^{\ddagger}$, Jennifer S. Haase ${ }^{\dagger}$ \\ and Bryan Clauss ${ }^{\dagger}$ \\ * School of Aeronautics and Astronautics \\ Purdue University \\ West Lafayette, IN 47907-2045 \\ $\dagger$ Department of Earth and Atmospheric Sciences \\ 550 Stadium Mall Dr \\ Purdue University \\ West Lafayette, IN 47907 \\ $\ddagger$ Jet Propulsion Laboratory \\ California Institute of Technology \\ Pasadena, CA 91109 \\ $\S$ NASA-Langley Research Center \\ Hampton, VA 23681
}

\begin{abstract}
In February-March 2009 NASA JPL conducted an airborne field campaign using the Passive Active L-band System (PALS) and the Ku-band Polarimetric Scatterometer (PolSCAT) collecting measurements of brightness temperature and near surface wind speeds. Flights were conducted over a region of expected high-speed winds in the Atlantic Ocean, for the purposes of algorithm development for salinity retrievals. Wind speeds encountered were in the range of 5 to $25 \mathrm{~m} / \mathrm{s}$ during the two weeks deployment. The NASA-Langley GPS delay-mapping receiver (DMR) was also flown to collect GPS signals reflected from the ocean surface and generate post-correlation power vs. delay measurements. This data was used to estimate ocean surface roughness and a strong correlation with brightness temperature was found. Initial results suggest that reflected GPS signals, using small low-power instruments, will provide an additional source of data for correcting brightness temperature measurements for the purpose of sea surface salinity retrievals.
\end{abstract}

\section{INTRODUCTION}

The thermal emission of seawater in microwave frequencies is a function of the dielectric constant, which in turn depends upon the sea surface salinity (SSS) and sea surface temperature (SST). L-band brightness temperature has long been studied for the remote sensing of SSS[1][2] through the use of this principle. Brightness temperature, however, is also strongly dependent on the surface roughness[3][4] and this dependence must be removed or corrected first, to retrieve

This research was supported by NASA grant NNX08A087G, "Comparison of GNSS-R Ocean Roughness Measurements with Radiometric and Scattering Models at L-band and S-band", Justin Voo was supported by the NASA Earth and Space Science Fellowship (NESSF) Program grant NNX07A049H to conduct a portion of this work at the Jet Propulsion Laboratory. The work described in this paper that was performed by the Jet Propulsion Laboratory, California Institute of Technology, was carried out under a contract with the National Aeronautics and Space Administration. salinity measurements from microwave radiometry. In most cases, this correction is made using auxiliary measurements of surface roughness, such as those provided by an active radar (scatterometer).

Bistatic radar, using signals from Global Navigation Satellite Systems (GNSS) as illumination sources, has also been shown to produce measurements of ocean surface roughness[5][6][7]. The global availability of these rangecoded signals, transmitted on L-band frequencies $(1.575 \mathrm{GHz})$ very near to the bands utilized for microwave radiometry, suggest them as potentially useful source for the roughness correction in microwave radiometry. This application has been proposed and studied theoretically[8][9][10]. In this paper, we will present experimental results comparing the brightness temperature correction using this technique (hereafter referred to as GNSS-R) with those from the $\mathrm{Ku}$-band polarimetric measurements from the PolSCAT instrument.

The GNSS-R technique has several attractive features, in addition to the fortuitous L-band frequency allocation for satellite navigation. Through re-utilization of existing signals with a passive receiver, the instrumentation would be considerably smaller and use less power than an active radar. Forwardscatter geometry results in a stronger received signal and the "process gain" provided through de-spreading the spread spectrum signal results in an increase in signal to noise ratio. These effects enable taking useful measurements with a lowgain antenna. Sensitivity to the surface roughness is present in the shape of the Delay-Doppler map (DDM), generated by cross-correlating the reflected signal with a local signal replica. Estimation of the probability density function for surface slopes can be found by inversion of the DDM, which is insensitive to total scattered power and thus the measurement 
does not require accurate calibration.

Most previous work has used the dependence of brightness temperature on near surface wind speeds[11][12]. In the research presented in this paper, we will use the mean square slope (MSS), under the assumption of an isotropic, normal distribution of surface slopes, as the variable of interest, and derive an empirical relationship between the MSS obtained from inverting the GNSS-R measurements and brightness temperature $\left(\Delta T_{b}\right)$ measured by the PALS instrument. This derived relationship will then be used to invert the GNSS-R measurements to obtain estimates of $\Delta T_{b}$. These retrievals of $\Delta T_{b}$ will be compared with those obtained through applying the same approach to the POLSCAT wind speed measurements. Finally, we will attempt to integrate the GNSS-R MSS data with PolSCAT wind speed in a combined estimation of $\Delta T_{b}$.

The paper is organized as follows. Section II provides a review of the scattering model applied to the GNSS-R signal, showing the relationship between the slope PDF and the shape of the DDM. Section III describes the experiment conducted February-March 2009. Section IV describes the data reduction and section $\mathrm{V}$ presents the results showing the dependence of MSS on $\Delta T_{b}$. Conclusions and recommendations for further work are given in section VI.

\section{GNSS-R SigNAL MODEL}

A sufficient model for the direct line-of-sight signal received from each GPS satellite takes the following form

$$
s(t)=A(t-\tau) D(t-\tau) p(t-\tau) e^{\left[-2 \pi\left(f_{c}+f_{D}\right) t+\phi\right] j}+n(t)
$$

in which $p(t-\tau)$ is a baseband signal with binary phase-shift keyed (BPSK) modulation from a pesudo-random noise (PRN) code and $D(t)$ is the BPSK-modulated data message. For the $\mathrm{C} / \mathrm{A}$ code on the L1 channel (which was the signal used in all of the data collected in this experiment), $p()$ is a 1023-chip PRN code with a chipping rate of $1.023 \mathrm{MHz}$, giving a $1 \mathrm{~ms}$ period in the absence of Doppler. $f_{c}$ is the carrier frequency generated at the satellite transmitter $(1.575 \mathrm{GHz}$ for the L1 channel) and $f_{D}$ is the Doppler shift resulting from relative motion between the transmitter and receiver. $\phi$ is the phase which is proportional to the delay modulo $2 \pi . n(t)$ is noise with a bandwith approximately equal to the receiver front-end bandwidth. Estimation of the delay $(\tau)$ and Doppler $\left(f_{D}\right)$ is done by correlating the received signal with a model

$$
s_{m}(t)=p(t-\hat{\tau}) e^{-2 \pi\left(\hat{\left.f_{c}+\hat{f_{D}}\right) t j}\right.}
$$

and determining $\hat{\tau}$ and $\hat{f_{D}}$ which maximize this crosscorrelation.

This same correlation process can be applied to signals reflected from a random rough surface which are composed of an infinite distribution of reflected ray paths, each taking the form of (1) with a differential amplitude. The resulting expected value of this cross-correlation as a function of $\hat{\tau}$ and $\hat{f_{D}}$,

$$
\left\langle\left|\mathbf{Y}\left(\hat{\tau}, \hat{f_{D}}\right)\right|^{2}\right\rangle=\left\langle s(t) s_{m}^{*}\left(t-\hat{\tau}, \hat{f_{D}}\right)\right\rangle
$$

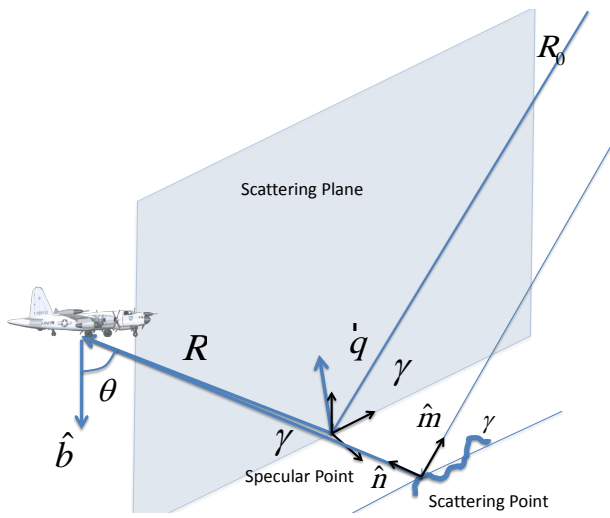

Fig. 1. Surface Scattering Coordinate Systems

is the DDM, which can be shown to be a surface integral dependent on the PDF of surface slopes. The relevant geometry for this model is shown in figure 1 in which the GPS satellite is assumed to be sufficiently far away such that the signal incident on the surface can be described by a plane wave at the same elevation angle, $\gamma$, as the satellite. Figure 1 defines a coordinate system fixed in the scattering plane, the earth is assumed to be flat at the specular point. $\vec{\rho}$ is the position vector of a point on the surface, as measured in this plane. The slope at each reflecting point on the surface is thus given by the vector $\vec{q}(\vec{\rho})=\frac{2 \pi}{\lambda} \hat{n}-\hat{m}$, where $\hat{m}$ and $\hat{n}$ are unit vectors in the incident and scattered directions, respectively.

Using the geometric optics approximation, which requires that the radius of curvature of surface features are much larger than the GPS wavelength, the DDM model, derived first in [8] is given by:

$$
\begin{aligned}
\left\langle|\mathbf{Y}(\tau, f)|^{2}\right\rangle=T_{i}^{2} \int & \int \frac{D^{2}(\vec{\rho}) \sigma_{0}(\vec{\rho})}{4 \pi R_{0}^{2}(\vec{\rho}) R^{2}(\vec{\rho})} \\
& \left|\chi\left(\tau-R_{0}(\tau)-R(\tau), f-f_{s}(\vec{\rho})\right)\right|^{2} d^{2} \vec{\rho}
\end{aligned}
$$

$T_{i}$ is the coherent integration time, $D(\vec{\rho})$ is the footprint function of the antenna, $R_{0}, R$ are distances to the transmitter and receiver respectively, and $\chi(\Delta \tau(\vec{\rho}), \Delta f(\vec{\rho}))$ is the ambiguity function of the transmitted GNSS signal (the correlation of (1) and (2)) where $f_{s}(\vec{\rho})$ is the doppler frequency at the scattering point. Dependence on the slope PDF enters through the bistatic scattering cross-section, $\sigma_{0}(\vec{\rho})$.

$$
\sigma_{0}(\vec{\rho})=\frac{\pi|F|^{2} q^{4}(\vec{\rho})}{q_{z}^{4}(\vec{\rho})} P_{\vec{\nu}}\left(-\frac{\vec{q}_{\perp}}{q_{z}}\right)
$$

where $F$ is the complex Fresnel reflection coefficient and $P_{\vec{\nu}}$ is the slope PDF. As shown in [9] this model may be rewritten as a convolution of $\Lambda^{2}$ with $p(\eta)$, the distribution of reflected power from the surface at delay $\eta$.

$$
p(\eta)=T_{i}^{2} \iint \frac{D(\vec{\rho}) \delta\left(\eta-R_{0}-R\right)}{4 \pi R_{0}^{2}(\vec{\rho}) R^{2}(\vec{\rho})} \sigma(\vec{\rho}) d^{2} \rho
$$

Surface slopes are generally assumed to follow a bivariate Gaussian distribution with extra terms to account for skewness and peakedness effects [13][14][15]. For this work we neglect the expanded terms and assume the slope distribution to be 
Gaussian and defined by the upwind and crosswind variances. Using a DDM for a fixed doppler allows retrieval of MSS without wind direction since the a one dimension slice of the DDM is not sensitive to the principal axis direction thus the total slope variance is estimated by assuming an isotropic slope distribution in the forward model.

\section{EXPERIMENT DESCRIPTION}

Experiments were conducted on 02 March 2009 flying on the NASA P-3 aircraft out of the Goose-Bay, Labrador airport to an area located at approximately $46 \mathrm{E}, 57 \mathrm{~N}$ over the Atlantic Ocean. Data from three instruments, PALS[16], POLSCAT[17] and the DMR [9][18] were used to record brightness temperature, wind speed from $\mathrm{Ku}$-band polarimetric backscatter, and the GNSS-R DDM. A brief description is provided for each instrument and the flight campaign. Section IV will describe the data reduction and the inter-comparison of these measurements.

\section{A. Delay Mapping Receiver}

Since initial development in 1997, a number of functionally identical GPS Delay Mapping Receivers (DMR) have been built by the NASA-Langley Research Center and used in various field campaigns and studies. These include the NASAU. S. Dept. of Agriculture Soil Moisture Experiments of 2002 (SMEX02) [19][20][ and 2003 (SMEX03)[21][22] over agricultural areas, and flights over the desert environment Jornada Range near Las Cruces, New Mexico The DMR has also been used by the U. S. Army's Cold Regions Research and Engineering Laboratory (CRREL) for surface snow and ice detection studies and has been flown onboard National Oceanic and Atmospheric Administration (NOAA) "Hurricane Hunter" aircraft for monitoring ocean surface wind speed [15][23]. In the DMR, a bank of PRN code correlators is utilized to simultaneously correlate the received surface-reflected signal over a range of 14 successive delay times (or delay bins). There is $1 / 2$ PRN code chip delay spacing between each correlator and the overall delay of the first correlator in the bank is set to ensure that the primary correlation peak (from signals near the specular point) is near the middle of the correlator bank (bin 7). Following code correlation, further instrument processing produces a set of 14 'power vs. [relative] delay' measurements, recorded at $1 / 10$ second intervals. Each correlation result is squared as part of instrument signal processing, and the set of squared correlations, $C_{j}=\left\langle\left|\mathbf{Y}\left(\hat{\tau_{j}}, \hat{f_{D}}\right)\right|^{2}\right\rangle j=[1, . .14]$, is recorded.

\section{B. PALS}

The PALS instrument includes a combined L-band radiometer and scatterometer, operating at $1.413 \mathrm{GHz}$ and 1.26 $\mathrm{GHz}$, respectively. It was built to investigate the benefits of combining passive and active microwave sensors for ocean salinity and soil moisture remote sensing. When it was built in 1999, it had one receiver at L-band and one receiver at S-band for either radar or radiometer. There were frontend microwave switches to sequentially select the antenna vertical and horizontal polarization ports to measure vertically and horizontally polarized radio emissions or radar echoes. To investigate the polarimetric observations of land or sea surfaces, the original S-band receiver was converted to another L-band receiver to enable simultaneous reception of vertical and horizontal polarizations. This conversion was completed in 2006-2007 prior to PALS' participation in the Cloud and Land Surface Interaction Campaign (CLASIC) in 2007[24][16]. The PALS radiometer electronics were also modified to include a microwave hybrid after the front-end low noise amplifiers in the $\mathrm{V}$ and $\mathrm{H}$ receivers to produce the brightness temperature measurements at +45 and -45 degree linear polarizations, which can be used to derive the third Stokes parameter measurement. To achieve good calibration stability, the PALS front-end electronics were temperature-controlled to $0.1 \mathrm{C}$. We also applied temperature control $(0.1 \mathrm{C})$ to the antenna with heater pads and temperature controller to ensure the stability of antenna gain. The PALS radiometer outputs include the vertically polarized $(\mathrm{V})$ brightness temperature $\left(T_{V}\right)$, horizontally polarized $(\mathrm{H})$ brightness temperature $\left(T_{H}\right)$ and the third Stokes parameter $(\mathrm{U})$. The PALS radar provides the normalized radar backscatter cross-section $\left(\sigma_{0}\right)$ for V-transmit/V-receive(VV), V-transmit/H-receive (HV), H-transmit/H-receive (HH), and $\mathrm{H}$-transmit/V-receive (VH).

\section{POLSCAT}

The POLSCAT instrument is a Ku-band (13.9 GHz) scatterometer[25][17]. It includes two-axis gimbals for a conically scanning, parabolic antenna at constant elevation angle, which is controllable from 0 (nadir) to 65 degrees. POLSCAT transmits at a pulse-repetition-interval (PRI) of 350 microseconds with alternating vertical $(\mathrm{V})$ and horizontal $(\mathrm{H})$ polarization from pulse to pulse. For each transmit pulse, POLSCAT detects the $\mathrm{V}$ and $\mathrm{H}$ polarization echoes simultaneously through two parallel receivers. This transmit and receiving design results in four polarization combinations, $\mathrm{VV}, \mathrm{HV}, \mathrm{HH}$, and $\mathrm{VH}$, for the detected radar echoes. The first letter of each polarization combination denotes the receive polarization and the second letter denotes the transmit polarization. More detailed characteristics of the POLSCAT electronics, including the calibration loop for transmit power and receiver gain calibration, can be found in[25][17].

\section{Data Collection Campaign}

Flights consisted of inbound and outbound paths and a variety of flight maneuvers including wing wags and constant roll turns. Aircraft altitude during the measurements was kept close to constant at approximately $3400 \mathrm{~m}$. The flight path is shown in figure 2. On March 2 PolSCAT measured wind speeds ranged from approximately 4.5 to $29.5 \mathrm{~m} / \mathrm{s}$ steadily increasing and decreasing over the inbound and outbound paths of the flight respectively. The DMR also recorded measurements of the DDM for subsequent retrieval of MSS, these results are shown in 4. Accurate aircraft orientation and positioning was provided by an Applanix GPS/INS system onboard the aircraft. This data allowed the full scattering geometry to be constructed for MSS estimation. The surface 


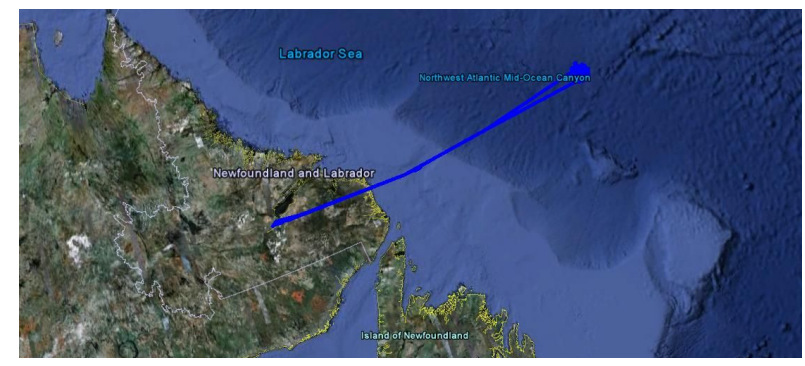

Fig. 2. Map of flight Path for March 2, 2009

salinity was assumed to be constant over the flight areas and the expected impact of surface salinity and surface temperature was negligible over the range of brightness temperatures observed.

\section{Data Procesing}

\section{A. Pre Processing}

The DDMs as measured by the DMR are the basic measurement used to retrieve surface slope statistics. The set of correlators used in the DMR, with fixed half-code chip spacing, is adjusted in instrument software to match the delay associated with the specular point. However, due to the fixed spacing changes in the path length of less than a half chip are not accounted for. Correcting the DDMs with the actual location of the specular point and using the known offset to the first correlator from the prompt correlator gives the relative distribution of power in the correct locations. An example of a re-aligned DDM is shown in figure 3, in this case the estimated MSS corresponding to the best fit was found to be .023 . The details can be found in [5].

\section{B. Surface Statistics Retrievals}

After aligning the correlation measurements, the power and corresponding delay is suitable for surface statistic retrieval. The estimation of MSS is done with a least squares approach to determine the slope standard deviation $(\sigma)$, a delay offset $\left(\tau_{0}\right)$, and a scale factor $\left(s_{\text {fact }}\right)$. The model DDM, generated from the scattering model described above, is fit to the measured data, using a 3-element parameter vector $\vec{p}=\left\{\sigma, \tau_{0}, s_{\text {fact }}\right\}$. Estimation of $\left(\tau_{0}\right)$ and $\left(s_{\text {fact }}\right)$ allow the model DDM to adjust for small uncertainties in delay as well as scaling to each individual block of measurements.

$\hat{\vec{p}}=\underset{\vec{p}}{\operatorname{argmin}}\left\{\left[Y^{2}(\tau, f)-Y_{M}^{2}(\tau, f ; \vec{p})\right]^{T}\left[Y^{2}(\tau, f)-Y_{M}^{2}(\tau, f ; \vec{p})\right]\right.$

RMS slope $(\sigma)$ was retrieved from each batch of 50 DDM's, producing an independent estimate using 5 seconds of data. The DDM with the estimated waveform is shown in figure 3.

\section{RESUlTS}

Total MSS $\left(\sigma^{2}\right)$ retrieved by performing the least squares estimation over the entire flight duration is shown in figure 5 along with piecewise curve fitting solutions, the range of

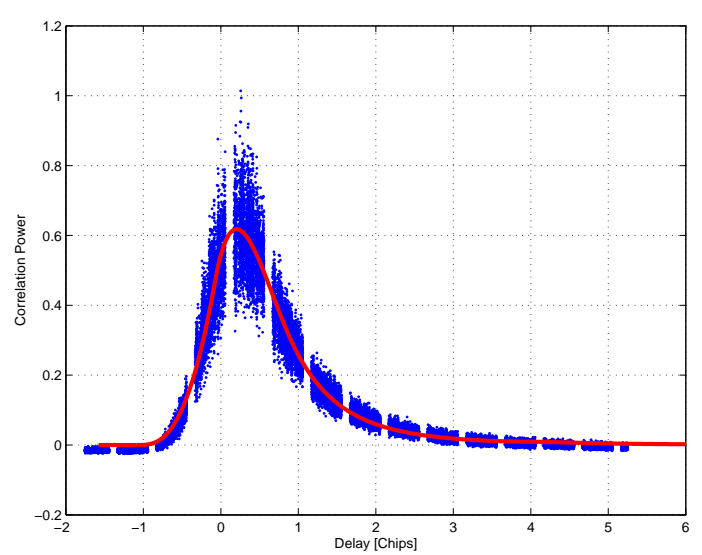

Fig. 3. Correlation Data with best fit

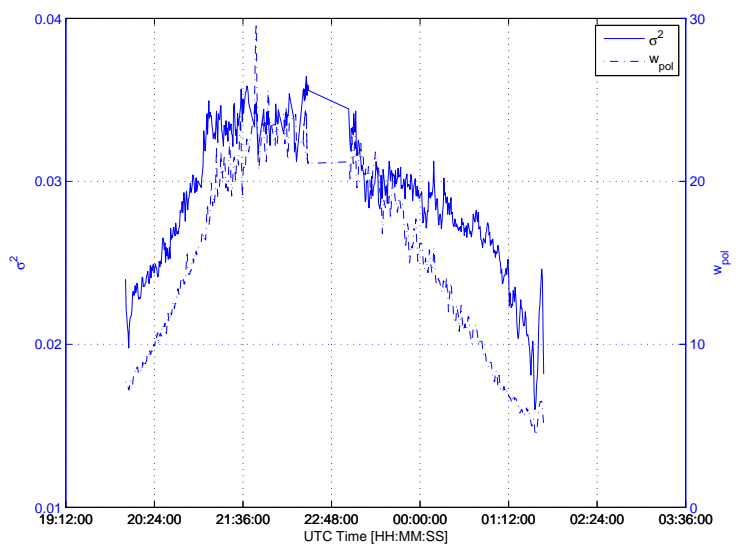

Fig. 4. $\quad \sigma^{2}$ Estimate for March 22009

$\sigma^{2}$ was approximately $.0155<\sigma^{2}<.0375$. The solution of $T_{b}$ to MSS was assumed to be of a form similar to that derived for wind speed [15], in this case a linear portion for small MSS and an exponential relation for large MSS $T_{b(H, V)}\left(\sigma^{2}\right)=a e^{b \sigma^{2}}+c e^{d \sigma^{2}}$ with coefficients $a, b, c, d$ to be determined.

In computing the exponential fits to the data certain points were excluded based on the cosine of the angle between the antenna boresight vector $(\hat{b})$ and the scattering vector at the specular point $\left(-\hat{n}_{s}\right)$ as shown in figure 1 . This was done due to possible nonuniform antenna gain pattern effects which are not taken into account in the forward model. This may also account for the varying groundtrack of the GNSS-R measurements relative to the aircraft fixed radiometer. This was computed by taking the dot product of the two vectors, the former of which was computed using the roll, pitch, and heading of the aircraft and is shown in figure 8. In figure 6 the residuals computed from an initial unweighted exponential fit to the data are plotted against the dot product value suggesting an empirical exclusion rule of $(\cos (\theta)<.851)$ to remove the largest errors. In figure 5 the least square curve fitting solution is shown where the influence of this rule can be seen, the points shown with a red $\mathrm{x}$ have been removed from consideration. The piecewise fits to MSS are given by 8 and 9. 

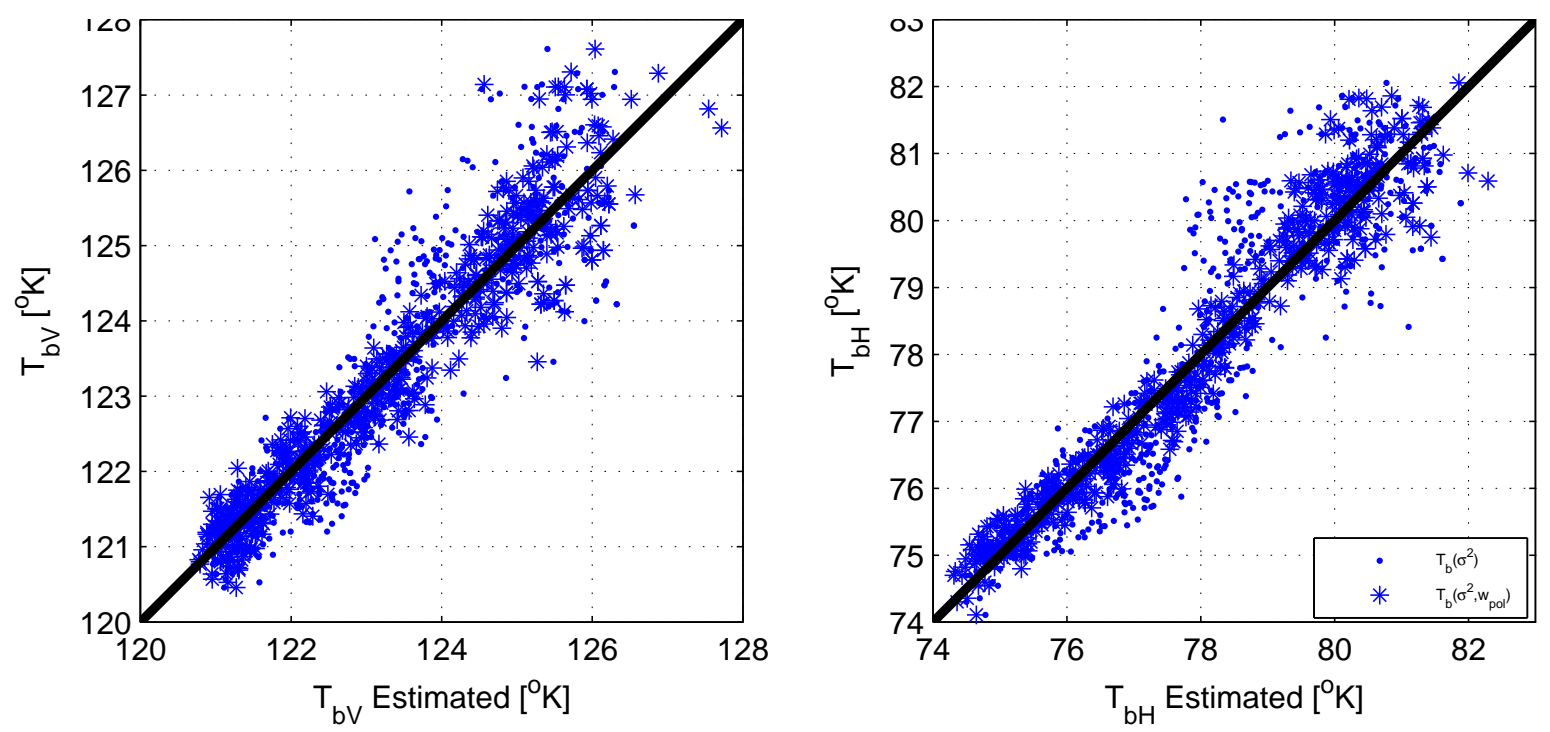

Fig. 7. $\quad T_{b}$ vs. $T_{b}$ stars represent a fused MSS and $w_{p o l}$ estimation, dots are estimations using MSS alone Left: $T_{b V}$ Right: $T_{b H}$
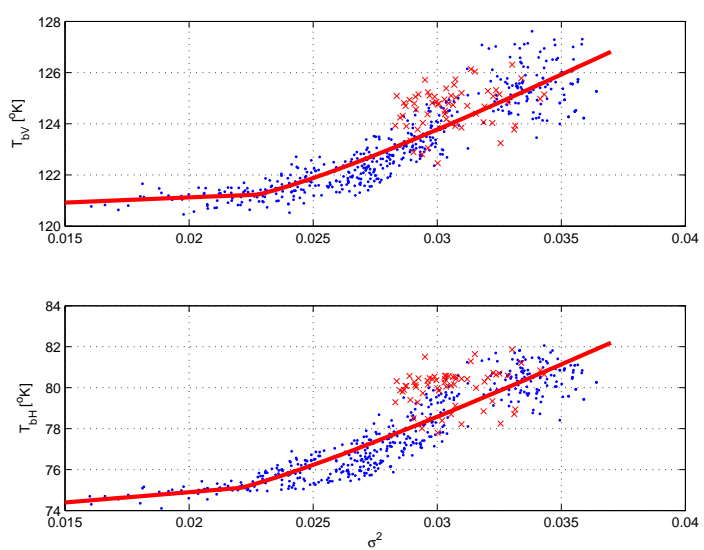

Fig. 5. Brightness Temperature with Piecewise Fit to $\sigma^{2}$ Top:Vertical Polarization Bottom:Horizontal Polarization
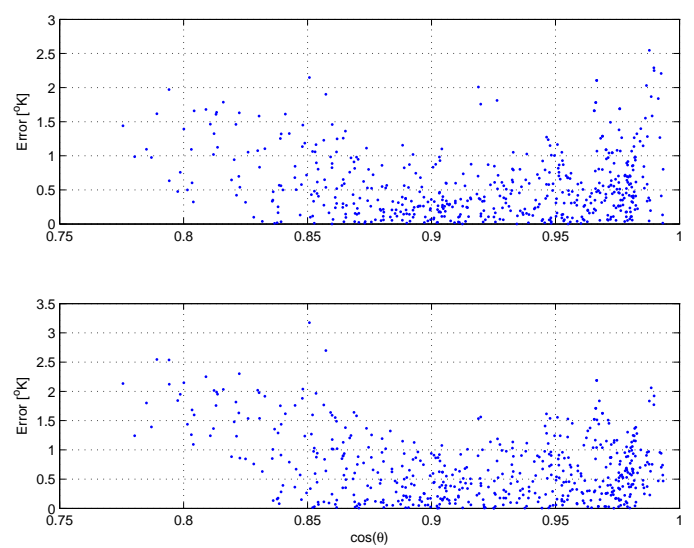

Fig. 6. Residuals vs. $\cos (\theta)$ Top: $T_{b V}$ Bottom: $T_{b H}$

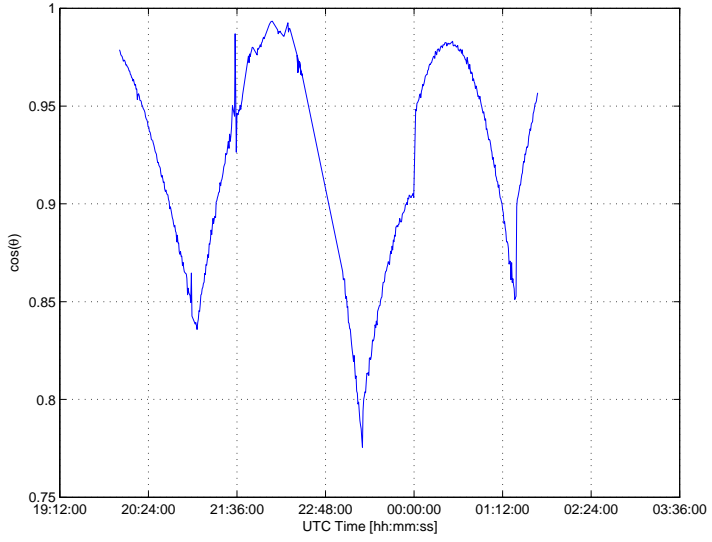

Fig. 8. $\cos (\theta)$ on March 2, 2009

$$
\begin{gathered}
T_{b V}= \begin{cases}40.4 \sigma^{2}+120.3 & \text { for } \sigma^{2} \leq .0228 \\
213.8 e^{-249.1 \sigma^{2}}+111.1 e^{3.6 \sigma^{2}} & \text { for } \sigma^{2}>.0228\end{cases} \\
T_{b H}= \begin{cases}107.1 \sigma^{2}+72.9 & \text { for } \sigma^{2} \leq .0221 \\
577.4 e^{-319.2 \sigma^{2}}+64.6 e^{6.5 \sigma^{2}} & \text { for } \sigma^{2}>.0221\end{cases}
\end{gathered}
$$

A multivariable solution was also found in both wind speed as measured by POLSCAT and MSS as measured by the DMR, a similar piecewise linear and exponential solution was used and the numerical coefficients in 10 and 11 were computed.

$T_{b V}=\left\{\begin{array}{l}.99 \sigma^{2}+.18 w_{p o l}+119.98 \quad \text { for } \sigma^{2} \leq .0228 \\ .288 w_{p o l}+33763.4 e^{.0012 \sigma^{2}}+70.9 e^{-878.3 \sigma^{2}} \\ +3.78 e^{-11.9 \sigma^{2} w_{p o l}}-33645.6 .0 \quad \text { for } \sigma^{2}>.0228\end{array}\right.$ 


\begin{tabular}{c|c}
\hline Fit Type & RMS Error $\left[{ }^{\circ} K\right]$ \\
\hline$T_{b V}\left(w_{p o l}\right)$ & 0.503131 \\
\hline$T_{b H}\left(w_{p o l}\right)$ & 0.463798 \\
\hline$T_{b V}\left(\sigma^{2}\right)$ & 0.688453 \\
\hline$T_{b H}\left(\sigma^{2}\right)$ & 0.768167 \\
\hline$T_{b V}\left(\sigma^{2}, w_{p o l}\right)$ & 0.481308 \\
\hline$T_{b H}\left(\sigma^{2}, w_{p o l}\right)$ & 0.462640 \\
\multicolumn{2}{|c}{ TABLE I } \\
SUMMARY OF RMS ERRORS
\end{tabular}

$T_{b H}=\left\{\begin{array}{l}-17.9 \sigma^{2}+.376 w_{p o l}+72.9 \quad \text { for } \sigma^{2} \leq .0221 \\ .388 w_{p o l}-550097.7 e^{-866.1 \sigma^{2}}+292.4 e^{-.051 \sigma^{2}} \\ +12.5 e^{-.04 \sigma^{2} w_{p o l}}-232 \quad \text { for } \sigma^{2}>.0221\end{array}\right.$

In this work the antenna gain pattern was modeled as having a $\cos ^{2}$ dependence on the off nadir angle, $D=\left(\hat{\hat{b}} n_{s}\right)$. In which $\hat{b}$ is along the antenna boresite and $-\hat{n}_{s}$ is in the directon of the specular point. Figure 6 shows the RMS error of the fit to $T_{b V}$ and $T_{b H}$ from MSS where there appears to be a dependency on the dot product between the antenna boresight vector $(\hat{b})$ and the scattering vector at the specular point $\left(-\hat{n}_{s}\right)$. Figure 7 presents a scatter plot for PALS measured $T_{b}$ vs the estimated $T_{b}$ based on the curve fitting solutions to MSS (red) and the multivariable fit (blue) in both MSS and wind speed as measured by PolSCAT, for reference the $45^{\circ}$ line is plotted in black.

\section{CONCLUSIONS AND Future WORK}

For each type of fit for $T_{b}$ the resulting RMS error is shown in table I. Data from this experiment suggests that the shape of the delay-doppler map generated from GNSS-R signals may be used to estimate roughness effects on brightness temperature retrievals from microwave radiometry. Furthermore, current methods of correcting brightness temperature measurements due to surface roughness may benefit from coincident measurements of ocean surface statistics from reflected GNSS signals. There appears to be some dependency of RMS error on $\theta$ indicating that a more accurate model for antenna gain or correction for different observation points between GNSS$\mathrm{R}$ and radiometer groundtracks. Furthermore, using a full 2-D DDM to retrieve upwind and crosswind $\sigma^{2}$ and wind direction may improve the estimations.

\section{REFERENCES}

[1] H.-J.C. Blume, B. M. Kendall, and J. C. Fedors. Measurement of ocean surface temperature and salinity via microwave radiometry. Bound. Layer Meteorol., 13:295-308, 1978.

[2] C. T. Swift and R. E. McIntosh. Considerations for microwave remote sensing of ocean surface salinity. Geoscience and Remote Sensing, IEEE Transactions on, GE-21:480-491, 1983.

[3] Y. Sasaki, I. Asanuma, K. Muneyama, G. Naito, and T. Suzuki. The Dependence of Sea-Surface Microwave Emission on Wind Speed, Frequency, Incidence Angle, and Polarization Over The Frequency Range of 1 to $40 \mathrm{GHz}$. Geoscience and Remote Sensing, IEEE Transactions on, GE-25(2), March 1987.

[4] A. Stogryn. The Apparent Temperature of the Sea at Microwave Frequencies. Antennas and Propagation, IEEE Transactions on, AP15(2), March 1967.

[5] J. L. Garrison, A. Komjathy, V. U. Zavorotny, and S. J. Katzberg. Wind speed measurement using forward scattered gps signals. IEEE Trans. Geosci. Remote Sensing, 40:50-65, January 2002.
[6] E. Cardellach, G. Ruffini, D. Pino, A. Rius, A. Komjathy, and J. L. Garrison. Mediterranean balloon experiment: Ocean wind speed sensing from the stratosphere using gps reflections. Remote Sensing of the Environment, 88:351-362.

[7] S. Gleason, S. Hodgart, Y. Sun, C. Gommenginger, S. Mackin, M. Adjrad, and M. Unwin. Detection and Processing of Bistatically Reflected GPS Signals From Low Earth Orbit for the Purpose of Ocean Remote Sensing. Geoscience and Remote Sensing, IEEE Transactions on, 43(6):1229-1241, 2005.

[8] V. U. Zavorotny and A. G. Voronovich. Scattering of gps signals from the ocean with wind remote sensing application. IEEE Trans. Geosci. and Remote Sensing, 38(2):951-964, March 2000.

[9] J. L. Garrison, S. J. Katzberg, and M. I. Hill. Effect of sea roughness on bistatically scattered range coded signals from global positioning system. Geophys. Res. Lett, 25:2257-2260, 1998.

[10] T. Elfouhaily, DR Thompson, and L. Linstrom. Delay-Doppler analysis of bistatically reflected signals from theocean surface: theory and application. Geoscience and Remote Sensing, IEEE Transactions on, 40(3):560-573, 2002.

[11] J. Hollinger. Passive Microwave Measurements of Sea Surface Roughness. Geoscience Electronics, IEEE Transactions on, 9(3), July 1971.

[12] S. H. Yueh, R. West, W. J. Wilson, F. K. Li, E. G. Njoku, and Y. RahmatSamii. Error sources and feasibility for microwave remote sensing of ocean surface salinity. IEEE Trans. Geosci. Remote Sensing, 39:10491060, May 2001.

[13] C. S. Cox and W. H. Munk. Measurements of the roughness of the sea surface from photographs of the sun's glitter. J. Opt. Soc. Amer, 44:838-850, 1954.

[14] T. T. Wilheit. A model for the microwave emissivity of the ocean's surface as a function fo wind speed. Geoscience Electronics, IEEE Transactions on, GE-17(4):244-249, 1979.

[15] S. Katzberg, O. Torres, and G. Ganoe. Calibration of Reflected GPS for Tropical Storm Wind Speed Retrievals. Geophysical Research Letters, 33(L18602), 2006.

[16] R. Bindlish, T. Jackson, S. Ruijing, M. Cosh, S. Yueh, and S. Dinardo. Combined Passive and Active Microwave Observations of Soil Moisture During CLASIC. IEEE Geoscience and Remote Sensing Letters, 6(4):644-648, 2009.

[17] S. Yueh, W. Wilson, and S. Dinardo. Polarimetric Radar Remote Sensing of Ocean Surface Wind. Geoscience and Remote Sensing, IEEE Transactions on, 40:793-800, 2002.

[18] J. L. Garrison and S. J. Katzberg. The Application of Reflected GPS Signals to Ocean and Wetland Remote Sensing. Proceedings of the 5th International Conference on Remote Sensing for Marine and Coastal Environments, 1:522-529, 1998.

[19] D. Masters, S. J. Katzberg, and P. Axelrad. Airborne GPS Bistatic Radar Soil Moisture Measurements During SMEX02. Proceedings of the IEEE Geoscience and Remote Sensing Symposium, 2003.

[20] S. J. Katzberg, O. Torres, M. S. Grant, and D. Masters. Utilizing calibrated GPS reflected signals to estimate soil reflectivity and dielectric constant: results from SMEX02. Remote Sensing of the Environment, 100:17-28, 2005.

[21] M. S. Grant and J. S. Katzberg. Combined GPS Reflected Signal and Visual Imagery for Unsupervised Clustering and Terrain Classification. Proceedings of the IEEE SoutheastCon, pages 370-377, 2004.

[22] M. S. Grant, S. T. Acton, and S. J. Katzberg. Terrain-Moisture Classification Using GPS Surface-Reflected Signals. IEEE Geoscience and Remote Sensing Letters, 4:41-45, 2007.

[23] S. J. Katzberg and J. Dunion. Comparison of reflected GPS wind speed retrievals with dropsondes in tropical cyclones. Geophys. Res. Letters, 36(L17602, doi:10.1029/2009GL039512), 2009.

[24] S. Yueh, S. Dinardo, S. Chan, E. Njoku, T. Jackson, and R. Bindlish. Passive and Active L-Band System and Observations during the 2007 CLASIC Campaign. IEEE International Geoscience and Remote Sensing Symposium, 2008.

[25] S. Yueh, S. Dinardo, A. Akgiray, R. West, D. Cline, and K. Elder. Airborne Ku-band Polarimetric Radar Remote Sensing of Terrestrial Snow Cover. Geoscience and Remote Sensing, IEEE Transactions on, 47(10):3347-3364, 2009. 